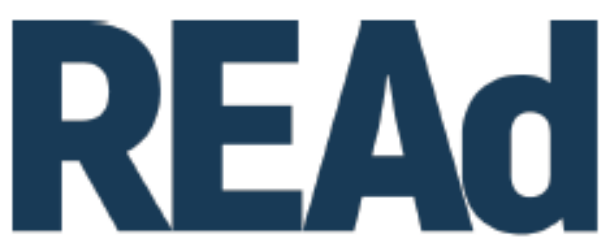

Revista Eletrônica de Administração

\title{
DESENVOLVIMENTO E VALIDAÇÃO DE UMA ESCALA DOS EFEITOS DA IDENTIFICAÇÃO CLUBÍSTICA EM TORCEDORES DE FUTEBOL ${ }^{1}$
}

\author{
George Alba ${ }^{2}$ \\ http://dx.doi.org/10.1590/1413-2311.314.103027
}

\section{RESUMO}

O presente trabalho busca desenvolver e validar uma escala que mensure os efeitos da identificação clubística em torcedores de futebol. Esses efeitos são explorados a partir de duas avenidas: atitudinal (comprometimento clubístico) e comportamental (lealdade e consumo clubístico). Foi aplicada uma survey em duas amostras, sendo uma online e não-probabilística para análise fatorial exploratória (477 respondentes) e outra presencial e probabilística para análise fatorial confirmatória (363 respondentes). Considerando que o futebol é um dos principais veículos de expressão dos brasileiros, a escala fornece uma abordagem útil à mensuração de elementos importantes inerentes ao consumo do esporte. $\mathrm{O}$ artigo contribui com a área de marketing, ampliando a capacidade científica para capturar a complexidade dos efeitos da identificação do torcedor de futebol com seu clube. A utilização prática do instrumento permite que os clubes possam ter diagnósticos mais consistentes e confiáveis das atitudes e comportamentos dos seus torcedores.

Palavras-chave: Marketing esportivo. Identificação clubística. Torcedores. Clubes de futebol. Escalas de marketing.

\section{DEVELOPMENT AND VALIDATION OF A SCALE FOR MEASURING THE EFFECTS OF TEAM IDENTIFICATION AMONG SOCCER FANS}

This paper aims to develop and validate a scale for measuring the effects of team identification among Brazilian soccer fans. These effects are explored from attitudinal (team commitment) and behavioral avenues (team loyalty and consumption). A survey was conducted in two samples, an online and non-probabilistic sample for exploratory factor analysis (507

\footnotetext{
${ }^{1}$ Recebido em 1552020, aceito em 2422021.

${ }^{2}$ Instituto Federal de Educação, Ciência e Tecnologia do Rio Grande do Sul e Universidade Federal do Rio Grande do Sul Porto Alegre- Brasil; http://orcid.org/0000-0002-4249-3996; george.alba@ufrgs.br.

(c) (1) \&) REAd | Porto Alegre - Vol. 27 - N.o 1 - Janeiro / Abril 2021 - p. 265-284.
} 
respondents) and a face-to-face and probabilistic sample for confirmatory factor analysis (363 respondents). Considering that soccer is one of the leading expressions of Brazilian life, the scale provides a useful tool for measuring elements inherent to sports consumption. The article contributes to marketing, advancing the scientific capacity to comprehend the complex effects of soccer fans identifying so strongly with their teams. The practical application of the instrument allows soccer teams to gather more reliable and consistent diagnoses of their fans' attitudes and behaviors.

Keywords: Sports marketing. Team identification. Supporters. Soccer clubs. Marketing scales.

\section{DESARROLLO Y VALIDACIÓN DE UNA ESCALA DE LOS EFECTOS DE LA IDENTIFICACIÓN CLUBISTA EN LOS AFICIONADOS AL FÚTBOL}

El presente trabajo tiene como objetivo desarollar y validar una escala que mida los efectos de la identificación con un cluben aficionados de fútbol brasileño. Estos efectos se explorarán a partir de dos vías: actitudinal (compromiso clubista) y conductual (lealtad y consumo clubista). Se aplicó una encuesta a dos muestras, una online y no probabilística para un análisis factorial exploratoria (477 encuestados) y otra presencial y probabilística para un análisis factorial confirmatoria (363 encuestados). Tomando en cuenta que el fútbol es uno de los principales vehículos de expresión de los brasileños, la escala proporciona un enfoque útil para la medición de elementos importantes inherentes al consumo del deporte. El artículo contribuye al área de marketing, ampliando la capacidad científica para capturar la complejidad de los efectos de identificación de los aficionados del fútbol con su equipo. La utilización práctica del instrumento permite a los clubes tener diagnósticos más consistentes y confiables de las actitudes y comportamientos de sus aficionados.

Palabras clave: Marketing deportivo. Identificación clubista. Aficionados. Clubes de fútbol. Escalas de marketing.

\section{INTRODUÇÃO}

"No futebol, o pior cego é o que só vê a bola."

Nelson Rodrigues

Torcedores gastam tempo, dinheiro e esforço consideráveis com seus clubes de futebol e muitas pessoas tornam-se torcedores tanto para construção de identidade, quanto para oportunidades de crescimento social (CRAWFORD, 2004). Quando um torcedor se identifica com um clube, este se torna uma de suas principais afiliações sociais, e acaba guiando seu comportamento e crenças em diversas áreas de seu cotidiano (GIBSON et al., 2002).

No Brasil, a relação entre os torcedores de clubes de futebol é a ilustração de uma situação em que o esporte está profundamente incorporado na vida, constituindo uma parte 
importante da cultura nacional (WACHELKE, 2008) e de um mercado consumidor relevante (LEONCINI; SILVA, 2005). A paixão pelo futebol é transmitida intergerações por meio de rituais e socialização entre consumidores (REALE; CASTILHOS, 2015) e a conexão dos torcedores com um clube tende a intensificar as intenções de consumir determinados produtos ou serviços a ele associados (SILVA et al., 2019).

A literatura acadêmica tem analisado diversas variáveis que envolvem a atitude e o comportamento dos fãs de esporte. Elas incluem a influência de família e amigos (WAKEFIELD, 1995, REALE; CASTILHOS, 2015), o interesse pela equipe e pelo esporte (MAHONY et al., 2002; ROBINSON et al. 2004), o valor de entretenimento (FUNK et al., 2002, SILVA; LAS CASAS, 2017), a lealdade e o envolvimento do torcedor (FUNK; JAMES, 2001; MAHONY et al., 2000; YOSHIDA et al. 2015), bem como a identificação com a equipe (FISHER; WAKEFIELD, 1998; MAHONY et al., 2002; MATSUOKA et al., 2003).

Para um cultivo eficiente da base de torcedores e da participação de mercado, o desenvolvimento de ferramentas de avaliação efetivas (BISCAIA et al., 2016) e identificação com os torcedores (KERR; WIJERATNE, 2020) é fundamental para os clubes esportivos. Apesar do Brasil ainda se encontrar em estágio inicial no estudo da gestão esportiva, a compreensão do comportamento e atitude dos torcedores deve estar na agenda de acadêmicos e gestores de futebol (FLEURY et al., 2016).

Este trabalho preenche uma lacuna teórica ao identificar elementos atitudinais e comportamentais relevantes à conexão entre torcedores de futebol e o clube o qual se identificam. Esses elementos são representados pelas dimensões: comprometimento, lealdade e consumo clubístico dos torcedores. O trabalho também preenche uma lacuna metodológica sob uma perspectiva de mensuração, uma vez que desenvolve uma escala que permite capturar manifestações internas e externas dos torcedores de futebol. A elaboração de um instrumento de mensuração substantivamente confiável e empiricamente válido é útil para que acadêmicos e práticos do marketing esportivo brasileiro ampliem a capacidade de diagnóstico sobre consequências da identificação dos torcedores com os clubes de futebol.

Stewart et al. (2003) sinalizaram que a pesquisa em consumo de esporte deve analisar de maneira holística alguns elementos da identificação com equipes. Especialmente no Brasil, a afiliação aos clubes de futebol parece ser um vetor de diversos fenômenos da vida moderna e determina a alocação de recursos financeiros, psicológicos e comportamentais de torcedores, como apontado por Silva e Las Casas (2017). Para Belk et al. (1989), muitos consumidores 
contemporâneos, como os torcedores de futebol, elevam o clube a um status religioso, o que torna a análise de seu comportamento ainda mais relevante.

O objetivo deste artigo é validar uma escala que mensure os efeitos da identificação clubística em torcedores de futebol. Esses efeitos serão explorados a partir de duas avenidas: atitudinal, por meio do comprometimento e comportamental, por meio da lealdade e do consumo clubístico. Considerando que o futebol é um dos principais veículos de expressão dos brasileiros (MEIHY, 1982), é relevante capturar a natureza da relação do torcedor de futebol com seu clube a partir de instrumentos de mensuração confiáveis.

Assim, o presente trabalho busca contribuir em três eixos: primeiro, constituir uma articulação teórica e estabelecer um conjunto de variáveis que permitam um melhor entendimento do consumo de futebol no Brasil; segundo, criar um instrumento capaz de capturar esses elementos com rigor científico e relevância prática e; por fim, avançar o debate na literatura sobre desenvolvimento de torcedores (KERR; WIJERATNE, 2020), estabelecendo a importância da mensuração e avaliação de diferentes dimensões da identificação do torcedor com seu clube de futebol.

\section{REVISÃO TEÓRICA}

A identificação com uma equipe esportiva, ou seja, a extensão em que uma pessoa sente uma conexão psicológica com uma determinada equipe (WANN; BRANSCOMBE, 1993), é um forte moderador das reações dos fãs sobre o desempenho de sua equipe (BERNACHEASSOLANT et al., 2007). Para Yoshida et al. (2015) e Kerr e Wijeratne (2020), o objetivo de adquirir e manter o apoio da base de fãs dedicados que consumirá o serviço da equipe de forma consistente é a essência da existência de um clube profissional. Não obstante, a conexão do torcedor com o clube intensifica atividades de consumo (SILVA et al., 2019). Lee e Zeiss (1980) constataram que fãs apresentam três formas de comprometimento: cognitivo, como o fã acumula conhecimento sobre o esporte e da equipe; atitudinal, como o fã acredita fortemente no esporte e na equipe e; comportamental, através de atos concretos, tais como comparecimento aos jogos. Além disso, a lealdade e comprometimento dos torcedores pode gerar, inclusive, atitudes e comportamentos positivos frente aos patrocinadores (BISCAIA et al., 2013).

Sutton et al. (1997) atribuíram a nomenclatura de "fãs adquiridos" àqueles que estão profundamente comprometidos com o seu clube. Esse fenômeno se manifesta de várias formas: no compromisso de filiação em longo prazo com um clube/equipe, em alocação de tempo e 
dinheiro, e na lealdade independentemente dos resultados. Funk e James (2006) indicam que tanto fatores pessoais, como socioculturais desempenham um papel proeminente no desenvolvimento de lealdade a uma equipe. Os motivos socioculturais referem-se às forças ambientais incorporadas no tecido da sociedade em que os consumidores de esportes vivem ou nas unidades sociais nos quais eles interagem (SMITH; STEWART, 2007).

Wann et al. (1996) observaram que as pessoas começaram e continuaram o apoio de uma equipe esportiva devido ao sucesso da equipe, sua localização geográfica, e apoio do grupo de pares para a equipe. Kolbe e James (2000) relataram que a decisão inicial de se tornar um torcedor é baseada na importância da família, dos amigos, de ir aos jogos e da socialização. Reale e Castilhos (2015) e James (2001) observaram que esses fatores influenciam o desenvolvimento inicial da preferência por uma equipe entre gerações, que revestem o consumo de futebol de poderes mágicos por meio de rituais (QUINTÃO et al., 2019).

As relações de identidade e conexão psicológica com equipes esportivas, muitas vezes, se traduzem em rituais de consumo (BELK et al., 1989). Os rituais são poderosos símbolos de significação que sustentam o comportamento aparentemente irracional dos torcedores (SMITH; STEWART, 2007), que não se limita apenas a emoções positivas (PRAYAG et al., 2020). No caso do futebol, é comum que os torcedores tenham maior interesse pelo clube que no esporte em si, assistindo e apoiando exclusivamente uma equipe. Já em um contexto de esportes individuais, como tênis ou boxe, não há equipe e, provavelmente, os espectadores estão mais motivados pelo seu interesse no esporte em si (KIM et al., 2008).

Muitos torcedores de futebol possuem certo grau de comprometimento apaixonado com o clube e tudo que está associado a ele (REDDEN; STEINER, 2000). Eles tendem a ser consumidores mais entusiasmados, e que não trocam de "fornecedor" jamais, e ilustram, metaforicamente (ou não), o "sonho" dos profissionais de marketing. A identificação dos torcedores com os clubes pode ser crítica para determinar a sustentabilidade das organizações esportivas (KERR; WIJERATNE, 2020) e podem oferecer oportunidades de ações de marketing independentes do desempenho da equipe. Os fãs de esporte comprometidos tendem a escutar programas especializados de rádio e televisão sistematicamente (LEE; ZEISS, 1980) e realizar leituras diárias a respeito da sua equipe (SMITH et al., 1981). Além disso, muitos torcedores fanáticos tendem a colecionar qualquer coisa que represente a equipe ou esporte, como roupas, pôsteres (FISKE, 1992), artigos de jornais e memorabília (HAYNES, 1993).

Para Bromberger et al. (1993), muitos torcedores são fanáticos por qualquer coisa relacionada ao seu clube e não perdem oportunidades em carregar o escudo do seu clube por 
onde vão. Estes torcedores apaixonados frequentam jogos e consomem mídias do clube de futebol mais intensamente (WAKEFIELD, 2016), além de estarem maduros para marketing de relacionamento, programas de fidelidade e maiores experiências de consumo (REDDEN; STEINER, 2000). Como alvo do marketing dos clubes, os torcedores clamam por estudos sistemáticos, e ainda, por um entendimento consistente de sua estrutura conceitual capaz de medir sua conexão com equipes ou interpretar o seu comportamento de consumo. Resultados dessa natureza devem fornecer uma série de benefícios analíticos para a manutenção (BISCAIA et al., 2016) e aumento da base de torcedores dos clubes (KERR; WIJERATNE, 2020).

\section{PROCEDIMENTOS METODOLÓGICOS}

O presente trabalho tem abordagem exploratória-descritiva de natureza quantitativa, com o objetivo de criar e validar uma escala para mensurar os efeitos da identificação clubística em torcedores de futebol. Assim, essa seção divide-se em duas partes: 1) desenvolvimento da escala, que abrange os aspectos metodológicos associados a elaboração do instrumento de mensuração e; 2) validação da escala, que abrange os aspectos metodológicos para validar empiricamente a escala em duas amostras de torcedores de clubes de futebol. Essencialmente, a primeira parte preocupa-se em definir o construto e suas dimensões, bem como a especificação dos itens do instrumento de mensuração, conforme procedimentos indicados por Rossiter (2002). Já a segunda parte demonstra como se deu o processo de amostragem e coletas de dados com torcedores, que ocorreram em maio e junho de 2018.

\subsection{DESENVOLVIMENTO DA ESCALA}

A escala dos efeitos da identificação clubística (EEIC) busca avaliar aspectos atitudinais e comportamentais relevantes à conexão entre torcedores e clubes de futebol. A elaboração do instrumento de coleta de dados foi conduzida de acordo com os procedimentos da teoria COARSE de Rossiter (2002), que é baseada na análise de conteúdo, estabelecida pela concordância de experts. Essa análise foi conduzida por dois pesquisadores de marketing experientes na utilização de escalas e possibilitou verificar o quão bem os componentes mensuráveis do construto representam o objeto de estudo em análise.

Foram elaboradas e adaptadas medidas para capturar as seguintes dimensões: 1) comprometimento clubístico, 2) lealdade clubística e, 3) consumo clubístico. A EEIC é 
composta por três dimensões e alguns dos seus itens foram derivados de trabalhos já existentes, como Mahony et al. (2000) e Bauer et al. (2008). Por se basear em trabalhos estrangeiros, a escala necessitou de uma avaliação de conteúdo e ambiente cautelosa, uma vez que os fãs de esporte brasileiros podem divergir dos outros países (WACHELKE, 2008).

Os itens da escala foram adaptados de outras escalas ou elaborados a partir de outras pesquisas da área, empregando os procedimentos metodológicos indicados por Rossiter (2002), que sugere que os construtos sejam definidos em termos de (1) objeto, (2) atributo, e (3) a unidade de avaliação. No caso da EEIC, não se pode conceber os efeitos da identificação em abstrato, sendo obrigatório um objeto focal relacionado ao atributo, neste caso um clube de futebol e uma entidade de avaliação, neste caso o torcedor do clube. Diante disso a definição conceitual do construto é a seguinte: "efeitos da identificação clubística nos torcedores de futebol", sendo o clube de futebol favorito, o objeto; os efeitos da identificação, o atributo e; o torcedor, a entidade de avaliação.

No Brasil, os torcedores entendem que um clube de futebol inclui elementos concretos e simbólicos no seu patrimônio, como a sede e história do clube, a camisa, o estádio, as cores, o hino, as insígnias, a mascote e os títulos (MORATO, 2005). Na abordagem de Rossiter (2002), o objeto "clube de futebol favorito" pode ser classificado como abstrato coletivo, dado que é uma coleção de objetos constituintes. Já o atributo "identificação com o clube" pode ser classificado como elicitante, considerando que ele consiste em características internas e manifestações exteriores. A entidade de avaliação "torcedor" pode ser classificada como um indivíduo, onde o que se busca é uma autoavaliação.

A escala busca avaliar estados e características individuais dos torcedores. Apesar de alguns destes estados ou características serem decorrentes de ações ou sentimentos que envolvam outras pessoas ou entidades, o foco reside na percepção individual desses fenômenos. Dado que o objeto focal é constituído por diversos objetos constituintes e os efeitos da identificação clubística podem ser considerados internos (comprometimento clubístico) ou um estado com manifestações externas (lealdade e consumo clubístico), os itens devem ser descritos como um conjunto de atividades distintas, mental ou física, que, como itens, são concretos e captam o domínio especificado.

Seguindo as orientações de Rossiter (2002), utilizou-se uma estrutura de quatro a oito itens por dimensão da escala. Os itens da escala correspondiam em afirmativas, onde os participantes deveriam indicar seu nível de concordância a partir de uma escala Likert de sete pontos, ancoradas em $1=$ discordo totalmente e $7=$ concordo totalmente. Para seleção e 
adaptação dos itens da dimensão comprometimento clubístico foram avaliadas as escalas Psychological Commitment to Team (PCT) de Mahony et al. (2000) e a escala Fan Loyalty de Bauer et al. (2008). Os itens foram classificados de acordo com o nível de associação com os componentes atitudinais do torcedor de clubes de futebol observados por Decrop e Derbaix (2010). Dois itens foram adaptados da escala de Mahony et al. (2000): seria difícil mudar minhas crenças sobre o [nome do clube]; eu aproveito qualquer ocasião para apoiar o [nome do clube], tanto em pensamentos quanto em emoções. Os outros dois itens foram adaptados da escala Bauer et al. (2008): não há nada que possa mudar o meu compromisso com o [nome do clube]; meu afeto com o [nome do clube] é independente dos resultados.

A dimensão lealdade clubística foi desenvolvida a partir da adaptação de quatro itens da dimensão comportamental da escala Fan Loyalty de Bauer et al. (2008). Alguns itens foram retirados e outros modificados, para permitir a utilização da escala em uma amplitude maior de torcedores de futebol, considerando a gênese do torcedor de futebol brasileiro. Seguindo as recomendações de Rossiter (2002), a escala necessitou ser simplificada, para que todos os itens avaliem o tempo presente. Originalmente, todos os itens são duplicados, avaliando um mesmo comportamento em dois tempos distintos (nesse caso, passado e futuro). A dimensão foi composta pelos seguintes itens: eu, frequentemente, assisto aos jogos do [nome do clube] na TV/internet/rádio; eu, frequentemente, acompanho informações sobre jogadores, técnicos e direção do [nome do clube] na mídia; eu, frequentemente, participo de discussões sobre o [nome do clube]; eu troco opiniões e sentimentos sobre o [nome do clube] com outras pessoas.

A dimensão consumo clubístico foi elaborada a partir dos achados de Decrop e Derbaix (2010). Para esses autores, o comportamento de consumo relacionado ao clube pode estar associado a diversas ações. Entretanto, um efeito tangível do consumo associado a identificação com o clube está relacionado com o quanto o torcedor consome itens da equipe em questão de quantidade de tempo e de dinheiro e quanto cria e personaliza objetos do clube. Sob esta perspectiva e segundo a classificação de Rossiter (2002), esta dimensão possui quatro atributos concretos, que necessitam apenas um item cada para efetiva mensuração. Decrop e Debaix (2010) indicam que a personalização de objetos está ligada à aplicação das cores, símbolos e escudos do clube nos objetos pessoais. Já a criação está associada à fabricação de bandeiras, flâmulas e faixas do clube. Os quatro itens foram elaborados a partir do trabalho de Decrop e Derbaix (2010): eu gasto uma quantidade considerável de tempo para colecionar itens relacionados ao [nome do clube]; eu gasto uma quantidade considerável de dinheiro para 
colecionar itens relacionados ao [nome do clube]; eu personalizo objetos com as cores, símbolos e escudos do [nome do clube]; eu crio bandeiras, flâmulas e faixas do [nome do clube].

Uma vez que as medidas da EEIC foram desenvolvidas ou adaptadas de trabalhos da língua inglesa, estes itens foram descritos em inglês e traduzidos para a língua portuguesa. A correta adaptação semântica e a manutenção do significado dos itens para o público pesquisado é de extrema relevância para a transmissão dos mesmos questionamentos que foram propostos originalmente. Para isso, seguiram-se os procedimentos estabelecidos por Pasquali (1998) para testes psicométricos. Utilizou-se o processo chamado back-translation, que consiste na tradução do instrumento original, depois na retradução para a língua de origem e por último, uma tradução final para a língua desejada. Nenhum dos tradutores havia tido contato com os instrumentos que traduziram, e particularmente, o terceiro tradutor não teve acesso a tradução do primeiro. Para satisfazer esses critérios foram utilizados três tradutores diferentes com fluência na língua inglesa e notável conhecimento na área de marketing. A primeira e a última tradução não mostraram diferenças significativas.

\subsection{VALIDAÇÃO DA ESCALA}

Uma pesquisa do tipo survey foi realizada em duas amostras independentes, como é recomendado no processo de validação de escalas utilizando análise fatorial exploratória e confirmatória. Para análise fatorial exploratória, se utilizou uma amostra online nãoprobabilística de 507 torcedores do Grêmio e do Internacional, coletada em maio de 2018. Para a análise fatorial confirmatória, se utilizou uma amostra probabilística, aplicada em 363 torcedores do Grêmio e Internacional em duas cidades do interior do Rio Grande do Sul, coletada em junho de 2018. Nas duas cidades, existem cerca de 26 mil moradores e o cálculo amostral delimitou em 363 coletas para um nível de confiança de 95\% e erro amostral de 5\% a partir de um arcabouço amostral de 6762 domicílios urbanos.

No período da coleta de dados para a análise fatorial confirmatória, os clubes ocupavam a mesma posição na tabela do campeonato brasileiro de futebol, que estava paralisado para a realização da Copa do Mundo FIFA 2018. A amostragem foi aleatória simples, uma vez que todas as residências não rurais das duas cidades tinham a mesma chance de serem sorteados para participar da pesquisa.

Dez assistentes de pesquisa realizaram a coleta e utilizaram os seguintes critérios para a validação dos indivíduos participantes da pesquisa: torcedor de um dos clubes Grêmio ou Inter 
e maior de 18 anos de idade. Os assistentes visitaram as casas das pessoas e convidaram para participar da pesquisa. Caso não houvesse participante apto para responder a pesquisa ou não houvesse ninguém presente, uma nova residência era sorteada. Após aceitarem participar da pesquisa, os torcedores responderam a um questionário que teve duração média de aplicação de cinco minutos.

A análise fatorial exploratória e confirmatória e a análise convergente seguiram as recomendações e os indicadores considerados satisfatórios conforme Hair et al. (2009). Já a análise discriminante seguiu as recomendações de Fornell e Larcker (1981). As análises de dados dos estudos foram realizadas por meio pacote estatístico IBM SPSS 23 e Amos 23.

\section{ANÁLISES}

\subsection{ANÁLISE FATORIAL EXPLORATÓRIA (EFA)}

Do total de 507 participantes, não houve missing values, uma vez que os respondentes só podiam avançar o questionário, quando todas as perguntas eram respondidas. Em termos de outliers, 30 observações foram excluídas por possuírem valores $\mathrm{p}<0,001$; ao considerar a distância de Mahalanobis. O total de questionários recebidos, eliminadas as observações atípicas (outliers), resultou em uma amostra de 477 casos, sendo 65,6\% gremistas, 62,3\% do sexo masculino e idade média de 32 anos. A mensuração de adequação da amostra pelo índice KMO indicou uma excelente condição para a utilização da EFA $(0,888)$. O teste de esferecidade de Bartlett, que examina a matriz de correlação indicou correlações significantes entre as variáveis $(\mathrm{p}<0,001)$, apresentando adequação da amostra à EFA. Quanto às comunalidades, todas tiveram valores acima de 0,60. Utilizou-se o método de extração de análise dos componentes principais e o método de rotação varimax, sendo que o critério de corte empregado foi de 0,55 . Os três fatores explicam $75 \%$ da variância, apresentando um resultado satisfatório para as três dimensões da escala. A matriz fatorial rotacionada apresenta cargas fatoriais entre 0,590 e 0,877, indicando ótimos níveis de significância prática. Os alfas de Cronbach da escala também apresentaram resultados satisfatórios.

\begin{tabular}{lcc} 
& Tabela 1 - Alfas de Cronbach & \\
Dimensão & Alfa de Cronbach & N de itens \\
Compromentimento clubístico & 0,809 & 4 \\
Lealdade clubística & 0,903 & 4 \\
Consumo clubístico & 0,903 & 4 \\
\hline \hline
\end{tabular}




\begin{tabular}{lccc} 
Tabela 2 - Análise fatorial exploratória (EFA) \\
\multicolumn{3}{c}{ Componente } \\
Item & 1 & 2 & $\frac{3}{, 771}$ \\
comp1 & & &, 785 \\
comp2 & & &, 780 \\
comp3 & & &, 590 \\
comp4 & & &, 845 \\
leal1 & &, 877 & \\
leal2 & &, 743 & \\
leal3 & &, 794 & \\
lea14 & & & \\
cons1 &, 845 & & \\
cons2 &, 849 & & \\
cons3 &, 818 & & \\
cons4 &, 802 & & \\
\hline \hline
\end{tabular}

\subsection{ANÁLISE FATORIAL CONFIRMATÓRIA (CFA)}

Dos 363 questionários respondidos, nove apresentaram missing values e foram removidos. Em termos de outliers, 17 observações foram excluídas por possuírem valores $\mathrm{p}<$ 0,001; ao considerar a distância de Mahalanobis. O total de questionários recebidos, eliminadas as observações atípicas (outliers), resultou em uma amostra de 337 casos, sendo 59,9\% gremistas, 60,8\% do sexo masculino e idade média de 34 anos. A CFA é utilizada neste trabalho para fornecer um teste confirmatório da teoria de mensuração. Diferentemente da análise fatorial exploratória, que forneceu a condução das variáveis para seus fatores de acordo com os dados, a CFA estabelece o quão bem a especificação dos fatores combina com a realidade (HAIR et al., 2009). A CFA foi conduzida na escala dos efeitos da identificação clubística (EEIC) por meio do software AMOS. As estimativas de caminho, que constituem um dos meios mais fáceis para identificar um problema potencial com uma teoria de mensuração, foram todas superiores a 0,7, que é considerado ideal. O modelo inicial sugeriu seis índices de modificação, que são calculados para cada relação possível que não é livre para ser estimada. As seis relações foram liberadas porque pertenciam a uma mesma dimensão da escala. Os índices de ajuste ao modelo de CFA foram satisfatórios. Já os resíduos padronizados, foram todos inferiores a |2,5|, o que não sugere problema. 


\begin{tabular}{lllllllll}
\hline \hline Modelo & $\chi^{2}$ & GL & $\chi^{2} / \mathrm{GL}$ & GFI & NFI & TLI & CFI & RMSEA \\
\hline Valores encontrados & 122,117 & 45 & 2,714 & 0,944 & 0,951 & 0,953 & 0,968 & 0,071 \\
Valores recomendáveis & & & $<3,00$ & $>0,900$ & $>0,900$ & $>0,900$ & $>0,900$ & $0,03 \sim 0,08$ \\
\hline \hline
\end{tabular}

\begin{tabular}{llcl}
\multicolumn{4}{c}{ Tabela 4 - Análise fatorial confirmatória (CFA) } \\
\hline \hline \multicolumn{3}{c}{ Dimensão } \\
Item & COMP & LEAL & CONS \\
comp1 &, 602 & & \\
comp2 &, 597 & & \\
comp3 &, 535 & & \\
comp4 &, 904 & & \\
leal1 & &, 685 & \\
leal2 & &, 734 & \\
leal3 & &, 731 & \\
leal4 & &, 848 &, 938 \\
cons1 & & &, 923 \\
cons2 & & &, 733 \\
cons3 & & &, 699 \\
cons4 & & & \\
\hline \hline
\end{tabular}

\subsection{VALIDADE CONVERGENTE E DISCRIMINANTE}

Para Hair et al. (2009), a quantia relativa de validade convergente entre medidas de itens, pode ser determinada pelas cargas fatoriais padronizadas, a variância extraída e a confiabilidade composta. As cargas fatoriais padronizadas foram analisadas e apresentaram resultados satisfatórios para todos os itens (acima de 0,5). A variância extraída apresentou valores superiores a 0,5, indicando convergência adequada. Já, a confiabilidade composta, que verifica a consistência interna dos indicadores, apresentou valores acima de 0,7. Já a validade discriminante sugere evidência de que um construto é único e captura fenômenos que outras medidas não conseguem (HAIR et al., 2009). Optou-se pela análise da validade discriminante pelo método de Fornell e Larcker (1981), que avalia a diferença entre a variância extraída (diagonal principal) de cada construto e o quadrado da correlação entre um par de construtos (fora da diagonal principal). Caso os quadrados da correlação entre construtos sejam inferiores as variâncias extraídas, pode se afirmar que há validade discriminante.

Tabela 5 - Confiabilidade composta e variância extraída (validade convergente) 


\begin{tabular}{lccccc}
\hline \hline Dimensão & $\begin{array}{c}(\Sigma \text { cargas } \\
\text { padronizadas })^{2}\end{array}$ & $\begin{array}{c}\Sigma \text { quadrados das } \\
\text { cargas padronizadas }\end{array}$ & $\begin{array}{c}\Sigma \text { erros de } \\
\text { mensuração }\end{array}$ & $\begin{array}{c}\text { Confiabilidade } \\
\text { composta }\end{array}$ & $\begin{array}{c}\text { Variância } \\
\text { extraída }\end{array}$ \\
\hline COMP & 6,959 & 1,822 & 2,178 & 0,762 & $45,56 \%$ \\
LEAL & 8,988 & 2,261 & 1,739 & 0,838 & $56,53 \%$ \\
CONS & 10,844 & 2,758 & 1,242 & 0,898 & $68,94 \%$ \\
\hline \hline
\end{tabular}

Tabela 6 - Validade discriminante

\begin{tabular}{llll}
\hline Dimensão & COMP & LEAL & CONS \\
COMP & $\mathbf{0 , 4 5 6}$ & & \\
LEAL & 0,352 & $\mathbf{0 , 5 6 5}$ & \\
CONS & 0,123 & 0,552 & $\mathbf{0 , 6 8 9}$ \\
& & & \\
\hline \hline
\end{tabular}

\section{DISCUSSÃO}

A escala dos efeitos da identificação clubística foi desenvolvida a partir de uma criteriosa definição conceitual de construto seguindo as recomendações de Rossiter (2002). Ao empregar os procedimentos da teoria COAR-SE, o objeto foi classificado como o clube de futebol favorito; os efeitos da identificação como o atributo e; o torcedor como a entidade de avaliação. A escala capturou as seguintes dimensões: 1) comprometimento clubístico, 2) lealdade clubística e, 3) consumo clubístico. Além de um desenvolvimento substantivamente confiável, as análises empregadas demonstraram a validade do instrumento de mensuração, preenchendo uma lacuna metodológica sobre a capacidade de capturar manifestações internas e externas da identificação clubística em torcedores de futebol brasileiros.

Independente do domínio do consumo, um dos principais objetivos dos gestores de marketing é encontrar clientes comprometidos, leais e que consumam efetivamente suas marcas, comunicando-se ativamente e ajudando na criação de valor. Naturalmente, analisar consumidores identificados com um clube de futebol é diferente de analisar consumidores identificados com uma empresa. A identificação dos torcedores com o clube é crítica para a sustentabilidade de uma organização esportiva (KERR; WIJERATNE, 2020), mesmo que esta possa suscitar tanto emoções positivas, quanto negativas (PRAYAG et al., 2020).

Os gestores de futebol necessitam de ferramentas analíticas e de avaliação para gerenciar a marca e cultivar relacionamentos sólidos e duradouros com seus torcedores (BISCAIA et al., 2016). Sob essa perspectiva, o desenvolvimento e validação de uma métrica capaz de capturar 
os efeitos da identificação clubística torna-se extremamente relevante, especialmente no Brasil, onde a paixão pelo futebol é transmitida intergerações (REALE; CASTILHOS, 2015).

Neste artigo, a abordagem de comprometimento e lealdade foram diferentes das frequentemente utilizadas na literatura de marketing tradicional. Os construtos lealdade e comprometimento clubístico foram baseados na literatura de consumo de esporte e marketing esportivo. A relevância desta observação reside no fato de que ainda existem obstáculos a serem superados no entendimento e na diferenciação do marketing esportivo para o marketing tradicional. Diferentemente de outros produtos e serviços, o esporte é frequentemente consumido irracionalmente. A lógica racional diz que se um produto continuamente não corresponde às expectativas, as pessoas vão parar de comprá-lo (CHADWICK, 2006). No esporte, esta lógica nem sempre se mantém. Por exemplo, os fãs que têm uma relação bem definida com um clube, vão apoiá-la, independentemente das vitórias ou derrotas (FISHER; WAKEFIELD, 1998), e ainda se envolverão em uma série de tarefas que beneficiam seu time favorito (YOSHIDA et al., 2015).

Em relação às contribuições relativas à mensuração, é importante salientar a adequação das escalas desenvolvidas em medir os construtos abordados no trabalho. As evidências de confiabilidade, validade de conteúdo, análise fatorial, validade convergente e validade discriminante sinalizam a adequação da escala desenvolvida para a mensuração dos efeitos da identificação clubística em torcedores de futebol. Este estudo teve uma orientação contextual no desenvolvimento da escala, o que garante um alinhamento adequado para mensuração de um fenômeno tão particular como a identificação clubística do torcedor de futebol brasileiro. Apesar do intenso debate sobre quais são os parâmetros mais importantes e a melhor forma de medi-los na literatura de marketing esportivo (KERR; WIJERATNE, 2020), ampliar utilização de ferramentas que possam proporcionar diagnósticos precisos ajudam a cultivar a base de torcedores (BISCAIA et al., 2016).

Tanto os pesquisadores de esporte (foco no torcedor), quanto os pesquisadores gerenciais (foco na gestão de clubes) se beneficiam da disponibilidade de métricas capazes de capturar aspectos atitudinais e comportamentais do torcedor. Análises efetivas da formação, das crenças e comportamentos dos consumidores de esporte podem promover resultados relevantes para o marketing (STEWART et al., 2003) e para os próprios consumidores. Ao conhecer melhor as atitudes e o comportamento dos seus torcedores, a gestão de marketing do clube se beneficia para elementos que vão além das vitórias e derrotas obtidos dentro de campo (FLEURY et al., 2016). A utilização da escala de efeitos da identificação clubística pode 
permitir que modelos de decisão sejam melhor explicados, a partir de um entendimento mais sólido da relação entre torcedores e seus clubes.

Duas limitações do trabalho merecem destaque. Em primeiro lugar, ambas as amostras utilizadas para a validação da escala foram de torcedores de apenas dois clubes de futebol (Grêmio e Internacional). É possível que os rituais de consumo de futebol associados a diferenças regionais possam impactar em algum nível as dimensões da escala. Mesmo que a paixão pelo clube de futebol seja um elemento da cultura nacional, os torcedores do sul do Brasil têm uma maior influência de costumes e tradições uruguaias e argentinas. Em segundo lugar, elementos situacionais referentes ao desempenho dos clubes no momento da coleta dos dados também podem ter influenciado os resultados. Porém, é importante ressaltar que ambos os times estavam com a mesma pontuação no campeonato brasileiro na fase de coleta de dados, o que pode ter atenuado potenciais diferenças dentro da amostra.

Por fim, considerando a importância que o futebol tem para os brasileiros e a crescente profissionalização dos clubes, existem oportunidades para que a academia de marketing ofereça métricas confiáveis para os gestores de futebol. Escalas como a desenvolvida neste trabalho podem ajudar a mapear a efetividade dos esforços de marketing dos clubes, além de segmentar os torcedores a partir de diferentes efeitos da identificação com o clube.

\section{REFERÊNCIAS}

BAUER, Hans H.; STOKBURGER-SAUER, Nicola E.; EXLER, Stefanie. Brand image and fan loyalty in professional team sport: A refined model and empirical assessment. Journal of sport Management, v. 22, n. 2, p. 205-226, 2008.

BELK, Russell W.; WALLENDORF, Melanie; SHERRY JR, John F. The sacred and the profane in consumer behavior: Theodicy on the odyssey. Journal of consumer research, $v$. 16, n. 1, p. 1-38, 1989.

BELK, Russell W. Possessions and the extended self. Journal of consumer research, v. 15, n. 2, p. 139-168, 1988.

BERNACHE-ASSOLLANT, Iouri; LACASSAGNE, Marie-Françoise; BRADDOCK, Jomills $\mathrm{H}$. Basking in reflected glory and blasting: Differences in identity-management strategies between two groups of highly identified soccer fans. Journal of Language and Social Psychology, v. 26, n. 4, p. 381-388, 2007.

BISCAIA, Rui et al. Sport sponsorship: The relationship between team loyalty, sponsorship awareness, attitude toward the sponsor, and purchase intentions. Journal of Sport Management, v. 27, n. 4, p. 288-302, 2013. 
BISCAIA, Rui et al. Investigating the role of fan club membership on perceptions of team brand equity in football. Sport Management Review, v. 19, n. 2, p. 157-170, 2016.

BROMBERGER, C., HAYOT, A., MARIOTTINI, J. (1993). “Allex I'O.M, forza Juve”: The passion for football in Marseille and Turin. In Redhead, S. (Ed.), The passion and the fashion: football fandom in the new Europe. Sydney: Avebury.

CHADWICK, Simon. Soccer marketing and the irrational consumption of sport.

International Journal of Sports Marketing \& Sponsorship, v. 7, n. 3, p. 153, 2006.

CRAWFORD, Garry. Consuming sport: Fans, sport and culture. Routledge, 2004.

DECROP, Alain; DERBAIX, Christian. Pride in contemporary sport consumption: a marketing perspective. Journal of the Academy of Marketing Science, v. 38, n. 5, p. 586$603,2010$.

FISHER, Robert J.; WAKEFIELD, Kirk. Factors leading to group identification: A field study of winners and losers. Psychology \& Marketing, v. 15, n. 1, p. 23-40, 1998. FISKE, John. The cultural economy of fandom. The adoring audience: Fan culture and popular media, p. 30-49, 1992.

FLEURY, Fernando A. et al. Efeito das Vitórias e Derrotas na Atitude do Torcedor de Futebol: um Estudo Envolvendo Garoto-Propaganda, Envolvimento e Fanatismo. Brazilian Business Review, v. 13, n. 4, p. 25, 2016.

FORNELL, Claes; LARCKER, David F. Structural equation models with unobservable variables and measurement error: Algebra and statistics. 1981.

FUNK, Daniel C.; JAMES, Jeff. The psychological continuum model: A conceptual framework for understanding an individual's psychological connection to sport. Sport management review, v. 4, n. 2, p. 119-150, 2001.

FUNK, Daniel C.; JAMES, Jeffrey D. Consumer loyalty: The meaning of attachment in the development of sport team allegiance. Journal of Sport Management, v. 20, n. 2, p. 189217, 2006.

FUNK, Daniel C.; MAHONY, Daniel F.; RIDINGER, Lynn L. Characterizing consumer motivation as individual difference factors: Augmenting the sports interest inventory (SII) to explain level of spectator support. Sport Marketing Quarterly, v. 11, n. 1, 2002.

GIBSON, Heather; WILLMING, Cynthia; HOLDNAK, Andrew. We're Gators... not just Gator fans: Serious leisure and University of Florida football. Journal of Leisure Research, v. 34, n. 4, p. 397-425, 2002.

HAIR, Joseph F. et al. Análise multivariada de dados. Bookman editora, 2009. 
HAYNES, Richard. Every man (?) a football artist: football writing and masculinity. S. Redhead, The passion and the Fashion: Football Fandom in the New Europe. Aldershot, Avebury, p. 55-76, 1993.

JAMES, Jeffrey D. The role of cognitive development and socialization in the initial development of team loyalty. Leisure Sciences, v. 23, n. 4, p. 233-261, 2001.

KERR, Anthony K.; WIJERATNE, Aaron J. 'Taking the pulse of a new football franchise': team identification and the Melbourne Heart FC in Australia's A-League. Soccer \& Society, p. 1-16, 2020.

KIM, Seungmo et al. An Analysis of Spectator Motives in an Individual Combat Sport: A Study of Mixed Martial Arts Fans. Sport Marketing Quarterly, v. 17, n. 2, 2008.

KOLBE, Richard H.; JAMES, Jeffrey D. An identification and examination of influences that shape the creation of a professional team fan. International Journal of Sports Marketing \& Sponsorship, v. 2, n. 1, p. 23-38, 2000.

LEE, Barrett A.; ZEISS, Carol A. Behavioral commitment to the role of sport consumer-an exploratory analysis. Sociology and Social Research, v. 64, n. 3, p. 405-419, 1980.

LEONCINI, Marvio Pereira; SILVA, Márcia Terra da. Entendendo o futebol como um negócio: um estudo exploratório. Gestão \& Produção, v. 12, n. 1, p. 11-23, 2005.

MAHONY, Daniel F.; MADRIGAL, Robert; HOWARD, Dennis A. Using the psychological commitment to team (PCT) scale to segment sport consumers based on loyalty. Sport marketing quarterly, v. 9, n. 1, p. 15, 2000.

MAHONY, Daniel F. et al. Motivational factors influencing the behaviour of J. League spectators. Sport Management Review, v. 5, n. 1, p. 1-24, 2002.

MATSUOKA, Hirotaka et al. Direct and interaction effects of team identification and satisfaction on intention to attend games. Sport Marketing Quarterly, v. 12, n. 4, p. 244$253,2003$.

MEIHY, José Carlos Sebe Bom. Para que serve o futebol. Futebol e cultura: coletânea de estudos, p. 11-19, 1982.

MORATO, Márcio Pereira. A dinâmica da rivalidade entre pontepretanos e bugrinos. Futebol, cultura e sociedade. Campinas: Autores Associados, p. 73-104, 2005.

PASQUALI, Luiz. Princípios de elaboração de escalas psicológicas. Revista de psiquiatria clínica, v. 25, n. 5, p. 206-213, 1998.

PRAYAG, Girish et al. Team identification, discrete emotions, satisfaction, and event attachment: a social identity perspective. Journal of Business Research, v. 112, p. 373-384, 2020. 
REDDEN, James; STEINER, Carol J. Fanatical consumers: towards a framework for research. Journal of consumer marketing, 2000.

QUINTÃO, Ronan Torres; PEREIRA, Verônica Fujise; BAÊTA, Rafael Antônio Santana. Consumo mágico: um estudo no contexto de torcedores de futebol. Revista Brasileira de Marketing, v. 18, n. 1, p. 17-28, 2019.

REALE, Getúlio; CASTILHOS, Rodrigo. Consumer socialization and intergenerational brand loyalty in the context of soccer. ACR North American Advances, 2015.

ROBINSON, Matthew J.; TRAIL, Galen T.; KWON, Hyungil. Motives and points of attachment of professional golf spectators. Sport Management Review, v. 7, n. 2, p. 167$192,2004$.

ROSSITER, John R. The C-OAR-SE procedure for scale development in marketing. International journal of research in marketing, v. 19, n. 4, p. 305-335, 2002.

SILVA, Edson Coutinho; LAS CASAS, Alexandre Luzzi. Sport fans as consumers: An approach to sport marketing. British Journal of Marketing Studies, v. 5, n. 4, p. 36-48, 2017.

SILVA, Thiago Bruno Jesus et al. Efeito do recall do patrocínio máster nos fatores de consumo de bens e serviços de um clube de futebol. RACE-Revista De Administração, Contabilidade E Economia, v. 18, n. 1, p. 113-136, 2019.

SMITH, Aaron CT; STEWART, Bob. The travelling fan: Understanding the mechanisms of sport fan consumption in a sport tourism setting. Journal of sport \& tourism, v. 12, n. 3-4, p. 155-181, 2007.

SMITH, Garry J. et al. A profile of the deeply committed male sports fan. Arena review, v. 5, n. 2, p. 26-44, 1981.

STEWART, Bob, SMITH, Aaron, NICHOLSON, Matthew. (2003). Sport consumption typologies: A critical review. Sport Marketing Quarterly, v. 12, n. 4, p. 206-16, 2003.

SUTTON, William A. et al. Creating and fostering fan identification in professional sports. Sport marketing quarterly, v. 6, p. 15-22, 1997.

WACHELKE, Joao Fernando Rech. Brazilian fans' social representations on soccer.(Representaciones sociales de los hinchas brasileños sobre fútbol). RICYDE. Revista Internacional de Ciencias del Deporte, v. 4, n. 13, p. 1-19, 2008.

WAKEFIELD, Kirk. Using fan passion to predict attendance, media consumption, and social media behaviors. Journal of Sport Management, v. 30, n. 3, p. 229-247, 2016.

WANN, Daniel L.; BRANSCOMBE, Nyla R. Sports fans: Measuring degree of identification with their team. International journal of sport psychology, 1993. 
WANN, Daniel L.; TUCKER, Kathleen B.; SCHRADER, Michael P. An exploratory examination of the factors influencing the origination, continuation, and cessation of identification with sports teams. Perceptual and motor skills, v. 82, n. 3, p. 995-1001, 1996.

YOSHIDA, Masayuki; HEERE, Bob; GORDON, Brian. Predicting behavioral loyalty through community: Why other fans are more important than our own intentions, our satisfaction, and the team itself. Journal of Sport Management, v. 29, n. 3, p. 318-333, 2015. 


\section{APÊNDICE: ESCALA DE EFEITOS DA IDENTIFICAÇÃO CLUBÍSTICA}

\begin{tabular}{|c|c|c|c|c|c|c|c|}
\hline \multicolumn{8}{|l|}{ Comprometimento clubístico } \\
\hline 1. Não há nada que possa mudar o meu compromisso com o meu clube. & 1 & 2 & 3 & 4 & 5 & 6 & 7 \\
\hline 2. Seria difícil mudar minhas crenças sobre o meu clube. & 1 & 2 & 3 & 4 & 5 & 6 & 7 \\
\hline 3. Meu afeto com o meu clube é independente dos resultados. & 1 & 2 & 3 & 4 & 5 & 6 & 7 \\
\hline $\begin{array}{l}\text { 4. Eu aproveito qualquer ocasião para apoiar o meu clube, tanto em pensamentos quanto em } \\
\text { emoções. }\end{array}$ & 1 & 2 & 3 & 4 & 5 & 6 & 7 \\
\hline \multicolumn{8}{|l|}{ Lealdade clubística } \\
\hline 5. Eu, frequentemente, assisto aos jogos do meu clube na TV/internet/rádio. & 1 & 2 & 3 & 4 & 5 & 6 & 7 \\
\hline $\begin{array}{l}\text { 6. Eu, frequentemente, acompanho informações sobre jogadores, técnicos e direção do meu } \\
\text { clube na mídia. }\end{array}$ & 1 & 2 & 3 & 4 & 5 & 6 & 7 \\
\hline 7. Eu, frequentemente, participo de discussões sobre o meu clube. & 1 & 2 & 3 & 4 & 5 & 6 & 7 \\
\hline 8. Eu troco opiniões e sentimentos sobre o meu clube com outras pessoas. & 1 & 2 & 3 & 4 & 5 & 6 & 7 \\
\hline \multicolumn{8}{|l|}{ Consumo clubístico } \\
\hline $\begin{array}{l}\text { 9. Eu gasto uma quantidade considerável de tempo para colecionar itens relacionados ao } \\
\text { meu clube. }\end{array}$ & 1 & 2 & 3 & 4 & 5 & 6 & 7 \\
\hline $\begin{array}{l}\text { 10. Eu gasto uma quantidade considerável de dinheiro para colecionar itens relacionados ao } \\
\text { meu clube. }\end{array}$ & 1 & 2 & 3 & 4 & 5 & 6 & 7 \\
\hline 11. Eu personalizo objetos com as cores, símbolos e escudos do meu clube. & 1 & 2 & 3 & 4 & 5 & 6 & 7 \\
\hline 12. Eu crio bandeiras, flâmulas e faixas do meu clube. & 1 & 2 & 3 & 4 & 5 & 6 & 7 \\
\hline
\end{tabular}

